\title{
On the Expected Discounted Penalty Function for a Markov Regime-Switching Insurance Risk Model with Stochastic Premium Income
}

\author{
Wenguang $Y u^{1,2}$ \\ ${ }^{1}$ School of Mathematics, Shandong University, Jinan 250100, China \\ ${ }^{2}$ School of Insurance, Shandong University of Finance and Economics, Jinan 250014, China \\ Correspondence should be addressed to Wenguang Yu; yuwg@mail.sdu.edu.cn
}

Received 3 December 2012; Accepted 30 January 2013

Academic Editor: Fuyi Xu

Copyright (C) 2013 Wenguang Yu. This is an open access article distributed under the Creative Commons Attribution License, which permits unrestricted use, distribution, and reproduction in any medium, provided the original work is properly cited.

\begin{abstract}
We consider a Markovian regime-switching risk model (also called the Markov-modulated risk model) with stochastic premium income, in which the premium income and the claim occurrence are driven by the Markovian regime-switching process. The purpose of this paper is to study the integral equations satisfied by the expected discounted penalty function. In particular, the discount interest force process is also regulated by the Markovian regime-switching process. Applications of the integral equations are given to be the Laplace transform of the time of ruin, the deficit at ruin, and the surplus immediately before ruin occurs. For exponential distribution, the explicit expressions for these quantities are obtained. Finally, a numerical example is also given to illustrate the effect of the related parameters on these quantities.
\end{abstract}

\section{Introduction}

In recent years, ruin theory under regime-switching model is becoming a popular topic. This model is proposed in Reinhard [1] and Asmussen [2]. Asmussen calls it a Markovmodulated risk model. And this model is also a generalization of the classical compound Poisson risk model. The primary motivation for this generalization is to enhance the flexibility of the model parameter settings for the classical risk process. The examples usually given are weather conditions and epidemic outbreaks, even though seasonality would play a role and can probably not be modeled by a Markovian regimeswitching model. Many papers on ruin probabilities and the expected discounted penalty function under the Markovian regime-switching risk model have been published. Some works in this area include $\mathrm{Ng}$ and Yang [3], $\mathrm{Li}$ and $\mathrm{Lu}$ [4], $\mathrm{Lu}$ and $\mathrm{Li}$ [5], Zhang [6], Zhu and Yang [7, 8], Yu [9], Dong et al. [10], Wei et al. [11], Elliott et al. [12], Ma et al. [13], Dong and Liu [14], Mo and Yang [15], Zhang and Siu [16], Li and Ren [17], and the references therein.

All of the researches mentioned above only take the constant interest force into consideration and do not take into account the impact of the change of the external environment. This provides the practical motivation to develop the ruin theory with stochastic interest force. In recent years, the ruin theory with stochastic interest force has attracted much attention in the actuarial science literature. See, for example, Ouyang and Yan [18], Cai [19], Zhao and Liu [20], Zhao et al. [21], and $\mathrm{Li}$ and $\mathrm{Wu}$ [22]. But these papers have only considered the question in which the interest force process, from the beginning to its end, has been described to be one process. Since the risk management of an insurance company is a longer-term program, these models cannot capture the feature that interest policies may need to change if economical or political environment changes. So it is natural to introduce the stochastic interest force regulated by the Markovian regime-switching process in insurance risk analysis. Zhang and Zhao [23] first consider the expected discounted penalty function in a classical risk model, in which the discount interest force process was modeled by the Markovian regime-switching process. Xie and Zou [24] study a compound binomial risk model with a constant dividend barrier under stochastic interest rates. Two types of individual claims, main claims and by-claims, are defined, where every 
by-claim is induced by the main claim and may be delayed for one time period with a certain probability. In the evaluation of the expected present value of dividends, the interest rates are assumed to follow a Markov chain with finite state space. Inspired by the work of Zhang and Zhao [23], in this paper, we generalize the risk model and assume that the claim process, the premium income process, and the stochastic interest force process are independently regulated by the Markovian regime-switching process.

The rest of this paper is organized as follows. In Section 2, the risk model and the stochastic interest force model are introduced. In Section 3, given the initial surplus and the initial environment state, the integral equation for the expected discounted penalty function is derived. In Section 4, for exponential distribution, we obtain the explicit expressions of the expected discounted penalty function. The results are illustrated by numerical examples in Section 5. Section 6 concludes the paper.

\section{The Risk Model and the Stochastic Interest Force Model}

Throughout the paper, we let $\left(\Omega, \mathscr{F},\left\{\mathscr{F}_{t}\right\}_{t \geq 0}, P\right)$ be a complete probability space with a filtration $\left\{\mathscr{F}_{t}\right\}_{t \geq 0}$ satisfying usual conditions containing all random variables and stochastic processes in our discussion.

Let $U(t)$ denote the surplus of an insurance company and be described as follows:

$$
U(t)=u+\sum_{k=1}^{N_{2}(t)} X_{k}-\sum_{k=1}^{N_{1}(t)} Y_{k}
$$

where $u \geq 0$ is the initial capital, $Y_{k}$ is the amount of the $k$ th claim, and $X_{k}$ is the amount of the $k$ th premium. $\left\{N_{1}(t) ; t \geq 0\right\}$ represents the number of claims occurring in $(0, t]$, and $\left\{N_{2}(t) ; t \geq 0\right\}$ represents the number of premium arrivals up to time $t$, both of which are described by the Markovian regime-switching process with intensity processes $\lambda_{1}(t)$ and $\lambda_{2}(t)$, respectively. Let the intensity processes $\lambda_{1}(t)$ and $\lambda_{2}(t)$ be homogeneous $n$-state and $m$-state Markovian process, respectively. The number of claims $\left\{N_{1}(t) ; t \geq 0\right\}$ is assumed to follow a Poisson distribution with parameter $\alpha_{1 i}$, and the corresponding claim amounts have distribution $G_{i}(z)$ when $\lambda_{1}(s)=\lambda_{1 i}, i=1,2, \ldots, n$ for $s \in(0, t]$. Similarly, the number of premium arrivals $\left\{N_{2}(t) ; t \geq 0\right\}$ has the Poisson distribution with parameter $\alpha_{2 j}$, and the corresponding premiums have distribution $F_{j}(x)$ when $\lambda_{2}(s)=$ $\lambda_{2 j}, j=1,2, \ldots, m$ for $s \in(0, t]$. We further assume that all states of the process $\lambda_{1}(t)$ communicate, which is also the case of the process $\lambda_{2}(t)$. The safety loading condition holds $E\left[\sum_{k=1}^{N_{2}(t)} X_{k}\right]>E\left[\sum_{k=1}^{N_{1}(t)} Y_{k}\right]$. Furthermore, we assume the processes $\left\{N_{1}(t) ; t \geq 0\right\},\left\{N_{2}(t) ; t \geq 0\right\},\left\{X_{i} ; i \geq 1\right\}$, and $\left\{Y_{i} ; i \geq 1\right\}$ are mutually independent.

Let $\eta_{i}=\eta_{i}\left(\lambda_{1 i}\right)$ be the rate at which the process $\lambda_{1}(t)$ leaves the state $\lambda_{1 i}$ and $p_{i k}$ the probability that it then goes to $\lambda_{1 k}$; that is, the intensity $\eta_{i k}$ of transition from $\lambda_{1 i}$ to $\lambda_{1 k}$ is given by

$$
\eta_{i k}= \begin{cases}\eta_{i} p_{i k} & \text { for } i \neq k, \\ -\eta_{i} & \text { for } i=k .\end{cases}
$$

Similarly, let $\bar{\eta}_{j}=\bar{\eta}_{j}\left(\lambda_{2 j}\right)$ be the rate at which the process $\lambda_{2}(t)$ leaves the state $\lambda_{2 j}$ and $\bar{p}_{j k}$ the probability that it then goes to $\lambda_{2 k}$; that is, the intensity $\bar{\eta}_{j k}$ of transition from $\lambda_{2 j}$ to $\lambda_{2 k}$ is given by

$$
\bar{\eta}_{j k}= \begin{cases}\bar{\eta}_{j} \bar{p}_{j k} & \text { for } j \neq k, \\ -\bar{\eta}_{j} & \text { for } j=k .\end{cases}
$$

The stochastic interest force function governed by the Markovian regime-switching process is defined by (Zhang and Zhao [23])

$$
r_{J}(t)=\delta_{J} t+\beta_{J} B(t)+\gamma_{J} P(t),
$$

where $J=\{J(t), t \geq 0\}$ is a homogeneous, irreducible, and recurrent Markovian process with finite state space $S=$ $\{1,2, \ldots, d\}$ with intensity matrix $\Lambda=\left(\varepsilon_{l k}\right)_{l, k=1}^{d}$, where $\varepsilon_{l l}:=$ $-\varepsilon_{l}$ for $l \in S$. As pointed out by Asmussen [2], in health insurance, sojourns of $\{J(t), t \geq 0\}$ could be certain types of epidemics, or, in automobile insurance, these could be weather types (e.g., icy, foggy, etc.). The state of interest is governed by $J(t)$. When the state of $J(t)$ is $l$, the interest force function is

$$
r_{l}(t)=\delta_{l} t+\beta_{l} B(t)+\gamma_{l} P(t), \quad l=1,2, \ldots, d,
$$

where $\delta_{l}, \beta_{l}$, and $\gamma_{l}$ are nonnegative constants, $B(t)$ is a standard Wiener process, and $P(t)$ is a Poisson process with parameter $\zeta$. Moreover, we also assume that $B(t), P(t)$ and $N(t)$ are independent of each other. Since stochastic fluctuation of interest cannot be large in reality, without loss of generality we might as well assume that

$$
\delta_{l}>\frac{1}{2} \beta_{l}^{2}+\zeta\left(e^{-\gamma_{l}}-1\right), \quad l=1,2, \ldots, d .
$$

Then the expected discounted penalty function with stochastic discount interest force driven by the Markovian regimeswitching process is defined as

$$
\begin{gathered}
\Phi_{i, j, l}(u) \\
=E\left[e^{-r_{l}\left(T_{u}\right)} w\left(U\left(T_{u}^{-}\right),\left|U\left(T_{u}\right)\right|\right) I\left(T_{u}<\infty\right) \mid U(0)\right. \\
\left.=u, \lambda_{1}(0)=\lambda_{1 i}, \lambda_{2}(0)=\lambda_{2 j}, J(0)=l\right],
\end{gathered}
$$

where $I(\cdot)$ is the indicator function, $T_{u}=\inf \{t: U(t)<0\}$ denotes the time of ruin, $U\left(T_{u}^{-}\right)$is the surplus immediately prior to ruin, $\left|U\left(T_{u}\right)\right|$ is the deficit at ruin, and $w(x, y)$ is a nonnegative bounded function on $[0, \infty) \times[0, \infty)$. We can interpret $\exp \left\{-r_{l}\left(T_{u}\right)\right\}$ as the "stochastic discount factor." The probability of ruin for $U(0)=u, \lambda_{1}(0)=\lambda_{1 i}$, and $\lambda_{2}(0)=\lambda_{2 j}$ is

$$
\begin{aligned}
& \Psi_{i, j}(u) \\
& \quad=\operatorname{Pr}\left(T_{u}<\infty \mid U(0)=u, \lambda_{1}(0)=\lambda_{1 i}, \lambda_{2}(0)=\lambda_{2 j}\right) .
\end{aligned}
$$


Obviously, $\Phi_{i, j}(u)$ can be reduced to $\Psi_{i, j}(u)$, if $r_{l}(t) \equiv 0$ and $w(x, y)=1$.

\section{The Integral Equation}

In this section, we derive the integral equation for the expected discounted penalty function.

Theorem 1. Suppose that the following conditions are satisfied:

(1) $\Phi_{i, j, l}(u),(i=1,2, \ldots n ; j=1,2, \ldots m ; l=1,2, \ldots d)$ is continuous with respect to $u$ on $[0,+\infty)$;

(2) $w(x, y)$ is continuous with respect to $x$;

(3) $\delta_{l}>(1 / 2) \beta_{l}^{2}+\zeta\left(e^{-\gamma_{l}}-1\right), l=1,2, \ldots, d$.

Then $\Phi_{i, j, l}(u)$ satisfies the following integral equation:

$$
\begin{aligned}
& {\left[\alpha_{1 i}+\alpha_{2 j}+\delta_{l}-\frac{1}{2} \beta_{l}^{2}-\zeta\left(e^{-\gamma_{l}}-1\right)\right] \Phi_{i, j, l}(u)} \\
& =\sum_{k=1}^{d} \varepsilon_{l k} \Phi_{i, j, k}(u)+\sum_{k=1}^{m} \bar{\eta}_{j k} \Phi_{i, k, l}(u) \\
& \quad+\sum_{k=1}^{n} \eta_{i k} \Phi_{k, j, l}(u) \\
& \quad+\alpha_{1 i}\left[\int_{0}^{u} \Phi_{i, j, l}(u-z) d G_{i}(z)\right. \\
& \left.\quad+\int_{u}^{\infty} w(u, z-u) d G_{i}(z)\right] \\
& +\alpha_{2 j} \int_{0}^{\infty} \Phi_{i, j, l}(u+x) d F_{j}(x)+o(h)
\end{aligned}
$$

where $F_{j}(x)$ and $G_{i}(z)$ are the distribution of the number of premiums and the claim amounts, respectively.

Proof. Consider $U(t)$ in an infinitesimal time interval $(0, h)$, and separate the seven possible cases as follows:

(1) no claim occurs in $(0, h)$, no change of claim state $i$ in $(0, h)$, no premium-arrival in $(0, h)$, no change of premium state $j$ in $(0, h)$, and no change of interest state $l$ in $(0, h)$, denoted by $A_{1}$;

(2) no claim occurs in $(0, h)$, no change of claim state $i$ in $(0, h)$, no premium-arrival in $(0, h)$, no change of premium state $j$ in $(0, h)$, and a change of interest state $l$ in $(0, h)$, denoted by $A_{2}$;

(3) no claim occurs in $(0, h)$, a change of claim state $i$ in $(0, h)$, no premium-arrival in $(0, h)$, no change of premium state $j$ in $(0, h)$, no change of interest state $l$ in $(0, h)$, denoted by $A_{3}$;

(4) one claim occurs in $(0, h)$, no change of claim state $i$ in $(0, h)$, no premium-arrival in $(0, h)$, no change of premium state $j$ in $(0, h)$, and no change of interest state $l$ in $(0, h)$, denoted by $A_{4}$;

(5) no claim occurs in $(0, h)$, no change of claim state $i$ in $(0, h)$, one premium-arrival in $(0, h)$, no change of premium state $j$ in $(0, h)$, and no change of interest state $l$ in $(0, h)$, denoted by $A_{5}$;

(6) no claim occurs in $(0, h)$, no change of claim state $i$ in $(0, h)$, no premium-arrival in $(0, h)$, a change of premium state $j$ in $(0, h)$, and no change of interest state $l$ in $(0, h)$, denoted by $A_{6}$;

(7) all other events with total probability $o(h)$.

By conditioning on the occurrence of claims, the change of claim state in $(0, h)$, the occurrence of premiums, the change of premium state in $(0, h)$, and the change of interest state in $(0, h)$, the expected discounted penalty function $\Phi_{i, j, l}(u)$ is equal to

$$
\begin{aligned}
& \Phi_{i, j, l}(u) \\
& =\sum_{k=1}^{6} E\left[e^{-r_{l}\left(T_{u}\right)} w\left(U\left(T_{u}^{-}\right),\left|U\left(T_{u}\right)\right|\right) I\left(A_{k}\right) \mid\right. \\
& \left.\quad U(0)=u, \lambda_{1}(0)=\lambda_{1 i}, \lambda_{2}(0)=\lambda_{2 j}, J(0)=l\right] \\
& +o(h) \\
& =I_{1}+I_{2}+I_{3}+I_{4}+I_{5}+I_{6}+o(h) .
\end{aligned}
$$

First, we consider $I_{1}$. We write $T_{u}$ as $h+T_{u} \circ \theta_{h}$ since $T_{u}>$ $h$. Because $r_{l}(t)$ has independent and stationary increments, $r_{l}(t), N_{1}(t)$, and $N_{2}(t)$ are the Markovian processes; so we have

$$
\begin{gathered}
I_{1}=E^{u}\left\{E \left[e^{-r_{l}\left(T_{u}\right)} w\left(U\left(T_{u}^{-}\right),\left|U\left(T_{u}\right)\right|\right) I\left(T_{u}<\infty\right)\right.\right. \\
\cdot I\left(N_{1}(h)=0, N_{2}(h)=0, \lambda_{1}(h)=\lambda_{1 i},\right. \\
\left.\left.\left.\lambda_{2}(h)=\lambda_{2 j}, J(h)=l\right) \mid \mathscr{F}_{h}\right]\right\} \\
=E^{u}\left\{e ^ { - r _ { l } ( h ) } I \left(N_{1}(h)=0, N_{2}(h)=0,\right.\right. \\
\left.\lambda_{1}(h)=\lambda_{1 i}, \lambda_{2}(h)=\lambda_{2 j}, J(h)=l\right) \\
\cdot E\left[e^{-\left[r_{l}\left(h+T_{u} \circ \theta_{h}\right)-r_{l}(h)\right]} w\left(U\left(\mathrm{~T}_{u}^{-}\right),\left|U\left(T_{u}\right)\right|\right)\right. \\
\left.\left.\times I\left(T_{u}<\infty\right) \mid r_{l}(h), U(h)\right]\right\}
\end{gathered}
$$




$$
\begin{aligned}
= & E^{u}\left\{e^{-r_{l}(h)} I\left(N_{1}(h)=0\right) I\left(N_{2}(h)=0\right) I\left(\lambda_{1}(h)=\lambda_{1 i}\right)\right. \\
& \left.\times I\left(\lambda_{2}(h)=\lambda_{2 j}\right) I(J(h)=l) \Phi_{i, j, l}(U(h))\right\} \\
= & \left(1-\alpha_{1 i} h+o(h)\right)\left(1-\eta_{i} h\right)\left(1-\alpha_{2 j} h+o(h)\right) \\
& \cdot\left(1-\bar{\eta}_{j} h\right)\left(1-\varepsilon_{l} h\right) e^{-\left(\delta_{l}-(1 / 2) \beta_{l}^{2}-\zeta\left(e^{-\gamma_{l}}-1\right)\right) h} \Phi_{i, j, l}(u) \\
= & {\left[1-\left(\alpha_{1 i}+\eta_{i}+\alpha_{2 j}+\bar{\eta}_{j}+\varepsilon_{l}+\delta_{l}-\frac{1}{2} \beta_{l}^{2}-\zeta\left(e^{-\gamma_{l}}-1\right)\right) h\right.} \\
& +o(h)] \Phi_{i, j, l}(u) .
\end{aligned}
$$

In the second case, an interest state change occurs; that is, the interest state changes from the state $l$ to $k(k \neq l)$ at switching time $v$, where $v$ lies in $[0, h)$. Hence, the interest discount factor at time $h$ should be written as $e^{-r_{k}(h-v)} \cdot e^{-r_{l}(v)}$, and $e^{-r_{l}\left(T_{u}\right)}$ should be revised as $e^{-r_{k}\left(T_{u}-h\right)} \cdot e^{-r_{l}(v)} \cdot e^{-r_{k}(h-v)}$. Therefore, by the same approach, we may obtain

$$
\begin{aligned}
& I_{2}=\sum_{k \neq 1} E^{u}\left\{I \left(N_{1}(h)=0, \quad N_{2}(h)=0,\right.\right. \\
& \lambda_{1}(h)=\lambda_{1 i}, \lambda_{2}(h)=\lambda_{2 j}, \\
& J(h)=k, 0<v \leq h) E \\
& \times\left[e^{-r_{k}\left(T_{u}-h\right)} e^{-r_{l}(v)} e^{-r_{k}(h-v)}\right. \\
& \cdot w\left(U\left(T_{u}^{-}\right),\left|U\left(T_{u}\right)\right|\right) \\
& \left.\left.\times I\left(T_{u}<\infty\right) \mid \mathscr{F}_{h}\right]\right\} \\
& =\sum_{k \neq l} E^{u}\left\{e^{-r_{k}(h-v)} e^{-r_{l}(v)} I\left(N_{1}(h)=0\right) I\left(N_{2}(h)=0\right)\right. \\
& \times I\left(\lambda_{1}(h)=\lambda_{1 i}\right) I\left(\lambda_{2}(h)=\lambda_{2 i}\right) \\
& \times I(J(h)=k, 0<v \leq h) \\
& \cdot E\left[e^{-r_{k}\left(T_{u} \theta_{h}\right)} w\left(U\left(T_{u}^{-}\right),\left|U\left(T_{u}\right)\right|\right)\right. \\
& \left.\left.I\left(T_{u}<\infty\right) \mid r_{k}(h), U(h)\right]\right\} \\
& =\sum_{k \neq l} \varepsilon_{l k} h\left(1-\alpha_{1 i} h+o(h)\right) \\
& \times\left(1-\eta_{i} h\right)\left(1-\alpha_{2 j} h+o(h)\right)\left(1-\bar{\eta}_{j} h\right) \\
& \cdot\left[1-\left(\delta_{k}-\frac{1}{2} \beta_{k}^{2}-\zeta\left(e^{-\gamma_{k}}-1\right)\right)\right. \\
& \times h+o(h)] \Phi_{i, j, k}(u) \\
& =\sum_{k \neq l} \varepsilon_{l k} \Phi_{i, j, k}(u) h+o(h) .
\end{aligned}
$$

Now we will turn to the third term in formula (10):

$$
\begin{aligned}
I_{3}= & \left(1-\alpha_{1 i} h+o(h)\right)\left(1-\alpha_{2 j} h+o(h)\right)\left(1-\bar{\eta}_{j} h\right) \\
& \cdot\left(1-\varepsilon_{l} h\right) e^{-\left(\delta_{l}-(1 / 2) \beta_{l}^{2}-\zeta\left(e^{-\eta_{l}}-1\right)\right) h} \sum_{k \neq i} \eta_{i k} \Phi_{k, j, l}(u) h \\
= & \sum_{k \neq i} \eta_{i k} \Phi_{k, j, l}(u) h+o(h) .
\end{aligned}
$$

For $I_{4}, I_{5}$, and $I_{6}$, we have

$I_{4}$

$$
\begin{aligned}
& =\left(1-\eta_{i} h\right)\left(1-\alpha_{2 j} h+o(h)\right)\left(1-\bar{\eta}_{j} h\right)\left(1-\varepsilon_{l} h\right) \\
& \qquad\left\{\int_{0}^{h} \alpha_{1 i} e^{-\alpha_{1 i} t}\right. \\
& \quad \times \int_{0}^{u} e^{-\left(\delta_{l}-(1 / 2) \beta_{l}^{2}-\zeta\left(e^{-\eta_{l}}-1\right)\right) t} \\
& \quad \times \Phi_{i, j, l}(u-z) d G_{i}(z) d t \\
& +\int_{0}^{h} \alpha_{1 i} e^{-\alpha_{1 i} t} \\
& \quad \times \int_{u}^{\infty} e^{-\left(\delta_{l}-(1 / 2) \beta_{l}^{2}-\zeta\left(e^{-\gamma_{l}}-1\right)\right) t} \\
& \left.\quad \times w(u, z-u) d G_{i}(z) d t\right\}+o(h) \\
& =\alpha_{1 i} h\left[\int_{0}^{u} \Phi_{i, j, l}(u-z) d G_{i}(z)\right. \\
& \left.+\int_{u}^{\infty} w(u, z-u) d G_{i}(z)\right]+o(h),
\end{aligned}
$$

$$
\begin{aligned}
I_{5}= & \left(1-\alpha_{1 i} h+o(h)\right)\left(1-\eta_{i} h\right)\left(1-\bar{\eta}_{j} h\right)\left(1-\varepsilon_{l} h\right) \\
& \cdot \int_{0}^{h} \alpha_{2 j} e^{-\alpha_{2 j} t} \\
\quad & \quad \int_{0}^{\infty} e^{-\left(\delta_{l}-(1 / 2) \beta_{l}^{2}-\zeta\left(e^{-\gamma_{l}}-1\right)\right) t} \Phi_{i, j, l}(u+x) d F_{j}(x) d t \\
= & \alpha_{2 j} h \int_{0}^{\infty} \Phi_{i, j, l}(u+x) d F_{j}(x)+o(h),
\end{aligned}
$$$$
I_{6}=\left(1-\alpha_{1 i} h+o(h)\right)\left(1-\eta_{i} h\right)\left(1-\alpha_{2 j} h+o(h)\right)
$$$$
\cdot\left(1-\varepsilon_{l} h\right) e^{-\left(\delta_{l}-(1 / 2) \beta_{l}^{2}-\zeta\left(e^{-\eta_{l}}-1\right)\right)} \sum_{k \neq j} \bar{\eta}_{j k} \Phi_{i, k, l}(u) h
$$$$
=\sum_{k \neq j} \bar{\eta}_{j k} \Phi_{i, k, l}(u) h+o(h) .
$$ 
It follows from (10)-(16) that

$$
\begin{aligned}
& \Phi_{i, j, l}(u) \\
& =\left[1-\left(\alpha_{1 i}+\eta_{i}+\alpha_{2 j}+\bar{\eta}_{j}+\varepsilon_{l}+\delta_{l}-\frac{1}{2} \beta_{l}^{2}-\zeta\left(e^{-\gamma_{l}}-1\right)\right)\right. \\
& \quad \times h+o(h)] \Phi_{i, j, l}(u) \\
& \quad+\sum_{k \neq l} \varepsilon_{l k} \Phi_{i, j, k}(u) h+\sum_{k \neq i} \eta_{i k} \Phi_{k, j, l}(u) h \\
& \quad+\alpha_{1 i} h\left[\int_{0}^{u} \Phi_{i, j, l}(u-z) d G_{i}(z)\right. \\
& \left.\quad+\int_{u}^{\infty} w(u, z-u) d G_{i}(z)\right] \\
& \quad+\alpha_{2 j} h \int_{0}^{\infty} \Phi_{i, j, l}(u+x) d F_{j}(x) \\
& \quad+\sum_{k \neq j} \bar{\eta}_{j k} \Phi_{i, k, l}(u) h+o(h) .
\end{aligned}
$$

Cancelling $\Phi_{i, j, l}(u)$, dividing both sides by $h$, and taking limit, the above equation reduces to (9).

Remark 2. If $n=m=d=1$ in (9), then let $\alpha_{1}=\alpha_{11}$, and $\alpha_{2}=\alpha_{21}$. This result can be reduced to a special case in which the interest process is described by stochastic interest; the premium process and the claim process are all compound Poisson processes; then the corresponding integral equation satisfied by the expected discounted penalty function is

$$
\begin{aligned}
& {\left[\alpha_{1}+\alpha_{2}+\delta-\frac{1}{2} \beta^{2}-\zeta\left(e^{-\gamma}-1\right)\right] \Phi(u)} \\
& =\alpha_{1}\left[\int_{0}^{u} \Phi(u-z) d G(z)+\int_{u}^{\infty} w(u, z-u) d G(z)\right] \\
& \quad+\alpha_{2} \int_{0}^{\infty} \Phi(u+x) d F(x) .
\end{aligned}
$$

Specially, if $n=m=d=1$ and $\beta=\gamma=0$, that is, $r(t)=\delta t$ in (9), then we have

$$
\begin{aligned}
& \left(\alpha_{1}+\alpha_{2}+\delta\right) \Phi(u) \\
& =\alpha_{1}\left[\int_{0}^{u} \Phi(u-z) d G(z)+\int_{u}^{\infty} w(u, z-u) d G(z)\right] \\
& \quad+\alpha_{2} \int_{0}^{\infty} \Phi(u+x) d F(x) .
\end{aligned}
$$

Remark 3. If $n=m=1, w(x, y)=1$, and $r_{J}(t)=0$ in (9), denoting nonruin probability $\varphi(u)=1-\Phi(u)$, then the integral equation in (9) is equivalent to the following:

$$
\begin{aligned}
& \left(\alpha_{1}+\alpha_{2}\right) \varphi(u) \\
& \quad=\alpha_{1} \int_{0}^{u} \varphi(u-z) d G(z)+\alpha_{2} \int_{0}^{\infty} \varphi(u+x) d F(x) .
\end{aligned}
$$

\section{The Explicit Results for Exponential Claim Distribution}

In this section, we consider the case that the claim amounts and premium numbers are exponentially distributed. We find that, in some specific settings, the expected discounted penalty function can be explicitly obtained. In most cases, it is difficult to obtain the precise expression of $\Phi_{i, j, l}(u)$, if we consider multiple states. Even if we narrow them to two states (i.e., $m=n=d=2$, at this point, we will get eight coupled equations), it would still be very hard for us to get the accurate expression of $\Phi_{i, j, l}(u)$. For the sake of simplicity only one state will be taken into account, that is, $m=n=d=1$. The purpose of this section is to get the explicit solution to prepare for the numerical calculation of the next section.

If $d=1$, that is, $r(t)=r_{J}(t)=\delta t+\beta B(t)+\gamma P(t)$, and if $w(x, y)=1$, then define $\phi(u)=E\left[e^{-r\left(T_{u}\right)} I\left(T_{u}<\right.\right.$ $\infty) \mid U(0)=u]$, which gives the Laplace transform of the time of ruin. Generally speaking, it is not easy to derive exact expression for $\phi(u)$. But in some special cases, such as the exponential distribution, we can obtain explicit form for the Laplace transform of the time of ruin

Theorem 4. If $n=m=d=1, F(x)=1-e^{-a x}, G(z)=1-e^{-b z}$, $a>0, b>0, \alpha_{2} / a>\alpha_{1} / b$, and $w(x, y)=1$, then for $u>0$

$$
\phi(u)=\frac{\alpha_{1}\left(a-\sigma_{1}\right)}{\left(\alpha_{1}+\alpha_{2}+A\right)\left(a-\sigma_{1}\right)-a \alpha_{2}} e^{\sigma_{1} u},
$$

where $\alpha_{1}=\alpha_{11}, \alpha_{2}=\alpha_{21}, A=\delta-(1 / 2) \beta^{2}-\zeta\left(e^{-\gamma}-1\right)$,

$$
\begin{aligned}
\sigma_{1}= & -\frac{b \alpha_{2}-a \alpha_{1}+(b-a) A}{2\left(\alpha_{1}+\alpha_{2}+A\right)} \\
& -\frac{1}{2} \sqrt{\left(-\frac{b \alpha_{2}-a \alpha_{1}+(b-a) A}{\alpha_{1}+\alpha_{2}+A}\right)^{2}+\frac{4 a b A}{\alpha_{1}+\alpha_{2}+A}}
\end{aligned}
$$

$<0$.

Moreover, when $r(t)=0$,

$$
\phi_{0}(u)=\frac{\alpha_{1}(a+b)}{b\left(\alpha_{1}+\alpha_{2}\right)} e^{\left(\left(a \alpha_{1}-b \alpha_{2}\right) /\left(\alpha_{1}+\alpha_{2}\right)\right) u} .
$$

Proof. By the methods of Yao et al. [25], let $\rho(u)=1-\phi(u)$. The change of $\rho(u)=1-\phi(u)$ in (9) leads to

$$
\begin{aligned}
& {\left[\alpha_{1}+\alpha_{2}+\delta-\frac{1}{2} \beta^{2}-\zeta\left(e^{-\gamma}-1\right)\right] \rho(u)} \\
& =\alpha_{1} \int_{0}^{u} \rho(u-z) b e^{-b z} d z+\alpha_{2} \int_{0}^{\infty} \rho(u+x) a e^{-a x} d x \\
& \quad+\delta-\frac{1}{2} \beta^{2}-\zeta\left(e^{-\gamma}-1\right) .
\end{aligned}
$$


TABLE 1: Exact values of $\phi(u)$ under different stochastic interest models.

\begin{tabular}{|c|c|c|c|c|c|c|c|c|c|}
\hline \multirow{3}{*}{$\beta$} & \multicolumn{9}{|c|}{$u=2$} \\
\hline & & $\delta=2.5$ & & & $\delta=2$ & & & $\delta=1.5$ & \\
\hline & $\gamma=0$ & $\gamma=0.5$ & $\gamma=1$ & $\gamma=0$ & $\gamma=0.5$ & $\gamma=1$ & $\gamma=0$ & $\gamma=0.5$ & $\gamma=1$ \\
\hline 0.0 & 0.011839 & 0.009891 & 0.008970 & 0.015540 & 0.012488 & 0.011144 & 0.021884 & 0.016600 & 0.014380 \\
\hline 0.1 & 0.011868 & 0.009912 & 0.008988 & 0.015587 & 0.012520 & 0.011140 & 0.021969 & 0.016653 & 0.014422 \\
\hline 0.2 & 0.011957 & 0.009976 & 0.009042 & 0.015730 & 0.012617 & 0.011218 & 0.022229 & 0.016814 & 0.014546 \\
\hline 0.3 & 0.012106 & 0.010084 & 0.009132 & 0.015973 & 0.012782 & 0.011352 & 0.022674 & 0.017087 & 0.014758 \\
\hline 0.4 & 0.012321 & 0.010239 & 0.009261 & 0.016325 & 0.013019 & 0.011543 & 0.023323 & 0.017483 & 0.015064 \\
\hline 0.5 & 0.012607 & 0.010444 & 0.009432 & 0.016797 & 0.013335 & 0.011798 & 0.024206 & 0.018016 & 0.015473 \\
\hline 0.6 & 0.012974 & 0.010705 & 0.009650 & 0.017408 & 0.013740 & 0.012123 & 0.025366 & 0.018707 & 0.016001 \\
\hline 0.7 & 0.013432 & 0.011029 & 0.009918 & 0.018181 & 0.014248 & 0.012529 & 0.026865 & 0.019585 & 0.016667 \\
\hline 0.8 & 0.013997 & 0.011426 & 0.010245 & 0.019151 & 0.014876 & 0.013028 & 0.028793 & 0.020691 & 0.017498 \\
\hline 0.9 & 0.014691 & 0.011907 & 0.010639 & 0.020363 & 0.015649 & 0.013637 & 0.031281 & 0.022081 & 0.018531 \\
\hline 1.0 & 0.015540 & 0.012488 & 0.011114 & 0.021884 & 0.016600 & 0.014380 & 0.034526 & 0.023837 & 0.019818 \\
\hline 1.1 & 0.016584 & 0.013193 & 0.011684 & 0.023807 & 0.017776 & 0.015289 & 0.038836 & 0.026075 & 0.021430 \\
\hline 1.2 & 0.017877 & 0.014048 & 0.012370 & 0.026271 & 0.019239 & 0.016406 & 0.044713 & 0.028972 & 0.023474 \\
\hline 1.3 & 0.019494 & 0.015096 & 0.013202 & 0.029489 & 0.021084 & 0.017792 & 0.053025 & 0.032802 & 0.026106 \\
\hline 1.4 & 0.021547 & 0.016392 & 0.014218 & 0.033797 & 0.023448 & 0.019534 & 0.065413 & 0.038014 & 0.029571 \\
\hline 1.5 & 0.024206 & 0.018016 & 0.015473 & 0.039761 & 0.026542 & 0.021763 & 0.085361 & 0.045384 & 0.034263 \\
\hline
\end{tabular}

TABLE 2: Exact values of $\phi_{1}(u)$ under different stochastic interest models.

\begin{tabular}{|c|c|c|c|c|c|c|c|c|c|}
\hline \multirow{3}{*}{$\beta$} & \multicolumn{9}{|c|}{$u=2$} \\
\hline & & $\delta=2.5$ & & & $\delta=2$ & & & $\delta=1.5$ & \\
\hline & $\gamma=0$ & $\gamma=0.5$ & $\gamma=1$ & $\gamma=0$ & $\gamma=0.5$ & $\gamma=1$ & $\gamma=0$ & $\gamma=0.5$ & $\gamma=1$ \\
\hline 0.0 & 0.015507 & 0.013395 & 0.012366 & 0.019332 & 0.016194 & 0.014730 & 0.025491 & 0.020392 & 0.018156 \\
\hline 0.1 & 0.015538 & 0.013418 & 0.012386 & 0.019380 & 0.016228 & 0.014758 & 0.025571 & 0.020444 & 0.018198 \\
\hline 0.2 & 0.015632 & 0.013489 & 0.012446 & 0.019523 & 0.016330 & 0.014843 & 0.025816 & 0.020603 & 0.018325 \\
\hline 0.3 & 0.015791 & 0.013608 & 0.012548 & 0.019767 & 0.016503 & 0.014986 & 0.026212 & 0.020874 & 0.018541 \\
\hline 0.4 & 0.016018 & 0.013778 & 0.012693 & 0.020118 & 0.016750 & 0.015191 & 0.026837 & 0.021263 & 0.018851 \\
\hline 0.5 & 0.016320 & 0.014003 & 0.012885 & 0.020587 & 0.017079 & 0.015463 & 0.027656 & 0.021786 & 0.019265 \\
\hline 0.6 & 0.016703 & 0.014288 & 0.013127 & 0.021190 & 0.017499 & 0.015809 & 0.028722 & 0.022458 & 0.019795 \\
\hline 0.7 & 0.017180 & 0.014639 & 0.013424 & 0.021947 & 0.018020 & 0.016237 & 0.030088 & 0.023306 & 0.020458 \\
\hline 0.8 & 0.017764 & 0.015066 & 0.013784 & 0.022887 & 0.018661 & 0.016760 & 0.031824 & 0.024363 & 0.021279 \\
\hline 0.9 & 0.018473 & 0.015579 & 0.014216 & 0.024051 & 0.019442 & 0.017393 & 0.034036 & 0.025677 & 0.022288 \\
\hline 1.0 & 0.019332 & 0.016194 & 0.014730 & 0.025491 & 0.020392 & 0.018156 & 0.036877 & 0.027314 & 0.023529 \\
\hline 1.1 & 0.020376 & 0.016931 & 0.015341 & 0.027286 & 0.021551 & 0.019079 & 0.040585 & 0.029370 & 0.025063 \\
\hline 1.2 & 0.021650 & 0.017816 & 0.016070 & 0.029549 & 0.022973 & 0.020198 & 0.045541 & 0.031985 & 0.026977 \\
\hline 1.3 & 0.023218 & 0.018884 & 0.016941 & 0.032446 & 0.024736 & 0.021566 & 0.052396 & 0.035374 & 0.029398 \\
\hline 1.4 & 0.025174 & 0.020184 & 0.017990 & 0.036242 & 0.026953 & 0.023257 & 0.062361 & 0.039883 & 0.032519 \\
\hline 1.5 & 0.027656 & 0.021786 & 0.019265 & 0.041372 & 0.029795 & 0.025377 & 0.077981 & 0.046101 & 0.036649 \\
\hline
\end{tabular}

By taking the derivative with respect to $u$ on the sides of (24), we have

$$
\begin{gathered}
{\left[\alpha_{1}+\alpha_{2}+\delta-\frac{1}{2} \beta^{2}-\zeta\left(e^{-\gamma}-1\right)\right] \rho^{\prime}(u)+\left(\alpha_{2} a-\alpha_{1} b\right) \rho(u)} \\
=\alpha_{2} a \int_{0}^{\infty} \rho(u+x) a e^{-a x} d x-\alpha_{1} b \int_{0}^{u} \rho(u-z) b e^{-b z} d z
\end{gathered}
$$

Differentiating the above equation with respect to $u$ again, we arrive at

$$
\begin{aligned}
& {\left[\alpha_{1}+\alpha_{2}+\delta-\frac{1}{2} \beta^{2}-\zeta\left(e^{-\gamma}-1\right)\right] \rho^{\prime \prime}(u)} \\
& +\left(\alpha_{2} a-\alpha_{1} b\right) \rho^{\prime}(u)+\left(a^{2} \alpha_{2}+b^{2} \alpha_{1}\right) \rho(u) \\
& \quad=\alpha_{2} a^{2} \int_{0}^{\infty} \rho(u+x) a \mathrm{e}^{-a x} d x+\alpha_{1} b^{2} \int_{0}^{u} \rho(u-z) b e^{-b z} d z
\end{aligned}
$$


TABLE 3: Exact values of $\phi_{2}(u)$ under different stochastic interest models.

\begin{tabular}{|c|c|c|c|c|c|c|c|c|c|}
\hline \multirow{3}{*}{$\beta$} & \multicolumn{9}{|c|}{$u=2$} \\
\hline & & $\delta=2.5$ & & & $\delta=2$ & & & $\delta=1.5$ & \\
\hline & $\gamma=0$ & $\gamma=0.5$ & $\gamma=1$ & $\gamma=0$ & $\gamma=0.5$ & $\gamma=1$ & $\gamma=0$ & $\gamma=0.5$ & $\gamma=1$ \\
\hline 0.0 & 0.005920 & 0.004946 & 0.004485 & 0.007770 & 0.006244 & 0.005557 & 0.010942 & 0.008300 & 0.007190 \\
\hline 0.1 & 0.005934 & 0.004956 & 0.004494 & 0.007794 & 0.006260 & 0.005570 & 0.010984 & 0.008327 & 0.007211 \\
\hline 0.2 & 0.005978 & 0.004988 & 0.004521 & 0.007865 & 0.006309 & 0.005609 & 0.011114 & 0.008407 & 0.007273 \\
\hline 0.3 & 0.006053 & 0.005042 & 0.004566 & 0.007986 & 0.006391 & 0.005676 & 0.011337 & 0.008543 & 0.007379 \\
\hline 0.4 & 0.006160 & 0.005119 & 0.004631 & 0.008162 & 0.006509 & 0.005772 & 0.011662 & 0.008741 & 0.007532 \\
\hline 0.5 & 0.006304 & 0.005222 & 0.004716 & 0.008399 & 0.006667 & 0.005899 & 0.012103 & 0.009008 & 0.007737 \\
\hline 0.6 & 0.006487 & 0.005353 & 0.004825 & 0.008704 & 0.006870 & 0.006062 & 0.012683 & 0.009354 & 0.008001 \\
\hline 0.7 & 0.006716 & 0.005515 & 0.004959 & 0.009091 & 0.007124 & 0.006265 & 0.013433 & 0.009793 & 0.008334 \\
\hline 0.8 & 0.006999 & 0.005713 & 0.005122 & 0.009575 & 0.007438 & 0.006514 & 0.014397 & 0.010346 & 0.008749 \\
\hline 0.9 & 0.007345 & 0.005953 & 0.005320 & 0.010181 & 0.007824 & 0.006819 & 0.015640 & 0.011041 & 0.009266 \\
\hline 1.0 & 0.007770 & 0.006244 & 0.005557 & 0.010942 & 0.008300 & 0.007190 & 0.017263 & 0.011918 & 0.009909 \\
\hline 1.1 & 0.008290 & 0.006596 & 0.005842 & 0.011903 & 0.008888 & 0.007644 & 0.019418 & 0.013038 & 0.010715 \\
\hline 1.2 & 0.008938 & 0.007024 & 0.006185 & 0.013136 & 0.009620 & 0.008203 & 0.022356 & 0.014486 & 0.011737 \\
\hline 1.3 & 0.009747 & 0.007548 & 0.006601 & 0.014744 & 0.010542 & 0.008896 & 0.026513 & 0.016401 & 0.013053 \\
\hline 1.4 & 0.010774 & 0.008196 & 0.007109 & 0.016898 & 0.011724 & 0.009767 & 0.032707 & 0.019007 & 0.014785 \\
\hline 1.5 & 0.012103 & 0.009008 & 0.007737 & 0.019881 & 0.013271 & 0.010881 & 0.042680 & 0.022692 & 0.017132 \\
\hline
\end{tabular}

from (24)-(26), we can obtain

$$
\begin{aligned}
& {\left[\alpha_{1}+\alpha_{2}+\delta-\frac{1}{2} \beta^{2}-\zeta\left(e^{-\gamma}-1\right)\right] \rho^{\prime \prime}(u)} \\
& \quad+\left[b \alpha_{2}-a \alpha_{1}+(b-a)\left(\delta-\frac{1}{2} \beta^{2}-\zeta\left(e^{-\gamma}-1\right)\right)\right] \rho^{\prime}(u) \\
& \quad-a b\left(\delta-\frac{1}{2} \beta^{2}-\zeta\left(e^{-\gamma}-1\right)\right) \rho(u) \\
& =-a b\left(\delta-\frac{1}{2} \beta^{2}-\zeta\left(e^{-\gamma}-1\right)\right) .
\end{aligned}
$$

Since $\delta-(1 / 2) \beta^{2}-\zeta\left(e^{-\gamma}-1\right)>0$, the corresponding homogeneous equation of the above differential equation with constant coefficients is

$$
\begin{aligned}
& \rho^{\prime \prime}(u)+\frac{b \alpha_{2}-a \alpha_{1}+(b-a) A}{\alpha_{1}+\alpha_{2}+A} \rho^{\prime}(u)-\frac{a b A}{\alpha_{1}+\alpha_{2}+A} \rho(u) \\
& =0 .
\end{aligned}
$$

Its characteristic equation is

$$
\sigma^{2}+\frac{b \alpha_{2}-a \alpha_{1}+(b-a) A}{\alpha_{1}+\alpha_{2}+A} \sigma-\frac{a b A}{\alpha_{1}+\alpha_{2}+A}=0,
$$

which has two real characteristic roots

$$
\begin{aligned}
\sigma_{1,2}= & -\frac{b \alpha_{2}-a \alpha_{1}+(b-a) A}{2\left(\alpha_{1}+\alpha_{2}+A\right)} \\
& \pm \frac{1}{2} \sqrt{\left(\frac{b \alpha_{2}-a \alpha_{1}+(b-a) A}{\alpha_{1}+\alpha_{2}+A}\right)^{2}+\frac{4 a b A}{\alpha_{1}+\alpha_{2}+A}} .
\end{aligned}
$$

Letting $\sigma_{1}<0, \sigma_{2}>0$ and noting that $\widetilde{\rho}(u)=1$ is one special solution of (27), hence,

$$
\rho(u)=C_{1} e^{\sigma_{1} u}+C_{2} e^{\sigma_{2} u}+1 .
$$

Noting that $u \rightarrow \infty, \rho(u) \rightarrow 1$, and $\sigma_{1}<0, \sigma_{2}>0$, so $C_{2}=0$, that is,

$$
\rho(u)=C_{1} e^{\sigma_{1} u}+1,
$$

which together with (24), implies

$$
\left(\alpha_{1}+\alpha_{2}+A\right)\left(1+C_{1}\right)=\alpha_{2} \int_{0}^{\infty}\left(C_{1} e^{\sigma_{1} x}+1\right) a e^{-a x} d x+A,
$$

so it follows that

$$
\phi(u)=\frac{\alpha_{1}\left(a-\sigma_{1}\right)}{\left(\alpha_{1}+\alpha_{2}+A\right)\left(a-\sigma_{1}\right)-a \alpha_{2}} e^{\sigma_{1} u} .
$$

When $r(t)=0,(27)$ is equivalent to

$$
\rho^{\prime \prime}(u)+\frac{b \alpha_{2}-a \alpha_{1}}{\alpha_{1}+\alpha_{2}} \rho^{\prime}(u)=0 .
$$

With the same argument, we can obtain

$$
\phi_{0}(u)=\frac{\alpha_{1}(a+b)}{b\left(\alpha_{1}+\alpha_{2}\right)} e^{\left(\left(a \alpha_{1}-b \alpha_{2}\right) /\left(\alpha_{1}+\alpha_{2}\right)\right) u} .
$$

Similarly, we can take many other suitable $w(x, y)$ in (7) when $n=m=d=1$. Then corresponding integral equations can be obtained. In general, it is not easy to derive exact solutions for the equations. However, when $F(x)$ and $G(z)$ are exponential, the explicit expression for the discounted expectation of the amount of surplus immediately before ruin occurs, and the deficit at ruin can be obtained. The process of proof is completely similar to that of Theorem 4 , and so it is omitted here. 
Remark 5. Let $w(x, y)=x$, and denote $\phi_{1}(u)=E\left[e^{-r\left(T_{u}\right)}\right.$ $U\left(T_{u}^{-}\right) I\left(T_{u}<\infty\right) \mid U(0)=u$ ], which can be considered as the discounted expectation of the surplus immediately before ruin occurs. If $n=m=d=1,(9)$ can be reexpressed as

$$
\begin{aligned}
\left(\alpha_{1}+\alpha_{2}+A\right) \phi_{1}(u) & \\
= & \alpha_{1}\left[\int_{0}^{u} \phi_{1}(u-z) d G(z)+\int_{u}^{\infty} u d G(z)\right] \\
& +\alpha_{2} \int_{0}^{\infty} \phi_{1}(u+x) d F(x) .
\end{aligned}
$$

Theorem 6. If $n=m=d=1, F(x)=1-e^{-a x}, G(z)=$ $1-e^{-b z}, a>0, b>0$, and $\alpha_{2} / a>\alpha_{1} / b$, then

$$
\phi_{1}(u)=D e^{\sigma_{1} u}-\frac{1}{b} e^{-b u}, \quad u \geq 0
$$

where

$$
D=\frac{\left(a-\sigma_{1}\right)\left[(a+b)\left(\alpha_{1}+\alpha_{2}+A\right)-a\right]}{b(a+b)\left[\left(\alpha_{1}+\alpha_{2}+A\right)\left(a-\sigma_{1}\right)-a \alpha_{2}\right]} .
$$

Remark 7. Let $w(x, y)=y$, and denote $\phi_{2}(u)=E\left[e^{-r\left(T_{u}\right)} \mid\right.$ $\left.U\left(T_{u}\right)\left|I\left(T_{u}<\infty\right)\right| U(0)=u\right]$, which can be considered as the discounted expectation of the deficit at ruin. If $n=m=d=1$, (9) can be re-expressed as

$$
\begin{aligned}
& \left(\alpha_{1}+\alpha_{2}+A\right) \phi_{2}(u) \\
& =\alpha_{1}\left[\int_{0}^{u} \phi_{2}(u-z) d G(z)+\int_{u}^{\infty}(z-u) d G(z)\right] \\
& +\alpha_{2} \int_{0}^{\infty} \phi_{2}(u+x) d F(x) .
\end{aligned}
$$

Theorem 8. If $n=m=d=1, F(x)=1-e^{-a x}, G(z)=1-e^{-b z}$, and $a>0, b>0, \alpha_{2} / a>\alpha_{1} / b$, then, for $u \geq 0$,

$$
\phi_{2}(u)=\frac{\left(a-\sigma_{1}\right) \alpha_{1}}{b\left(a-\sigma_{1}\right)\left[\alpha_{1}+\alpha_{2}+A\right]-a b \alpha_{2}} e^{\sigma_{1} u} .
$$

\section{Numerical Illustrations}

Taking into account the importance of the interest rates and the simplicity of discussion, we only consider the effect of stochastic interest on the $\phi(u), \phi_{1}(u)$, and $\phi_{2}(u)$. Let us give some data analysis about the theoretical results in formula (21), such that we can catch the effect information of the stochastic interest factors. We first need to determine the value of the parameters in formula (21). For convenience, we might as well suppose that $a=1, b=2, \alpha_{1}=1$, and $\alpha_{2}=1$. The constant interest force $\delta$ is assumed to be $1.5,2$, and 2.5; the coefficient $\beta$ starts at 0 and ends at 1.5 evenly spaced by the value 0.1 ; the coefficient $\gamma$ is valued at $0,0.5$, and 1 , the parameter $\zeta$ is supposed to be 1 . Based on the formula (21), the above assumptions, and MATLAB, we get the values of $\phi(u)$ under the different combinations of parameter values (see Table 1).

From Table 1, we can get the trend of changes of $\phi(u)$ when the other two parameters keep unchanged. (i) The $\phi(u)$ is increased steadily with increasing $\beta$ when $\delta$ and $\gamma$ are unchanged.

(ii) The $\phi(u)$ is increased with a small decrease of $\delta$ when $\beta$ and $\gamma$ are unchanged.

(iii) The $\phi(u)$ is decreased, if $\gamma$ increases when $\delta$ and $\beta$ are unchanged.

In the same way, we can also get similar conclusion for $\phi_{1}(u)$ (38) and $\phi_{2}(u)$ (41); so we omit the detailed description here. See Tables 2 and 3.

\section{Conclusions}

We have generalized the results in Zhang and Zhao [23]. We suppose that the premium income process, the occurrence of the claims, and the interest process are controlled by the Markov regime-switching process, respectively. We not only obtain the integral equations satisfied by the expected discounted penalty function under the stochastic interest force driven by the Markov regime-switching process, but also offer data analysis and direct interpretation based on the interest models for some special cases. These all provide insights into the effect of stochastic interest force on the expected discounted penalty function and show the importance of introducing stochastic interest force.

\section{Acknowledgments}

The author thanks the three anonymous referees for the thoughtful comments and suggestions that greatly improved the presentation of this paper. This work was supported by the National Natural Science Foundation of China (Grant no. 11171187 and Grant no. 10921101) National Basic Research Program of China (973 Program, Grant no. 2007CB814906) Natural Science Foundation of Shandong Province (Grant no. ZR2012AQ013 and Grant no. ZR2010GL013) and Humanities and Social Sciences Project of the Ministry Education of China (Grant no. 10YJC630092 and Grant no. 09YJC910004).

\section{References}

[1] J. M. Reinhard, "On a class of semi-Markov risk models obtained as classical risk models in a Markovian environment," Astin Bulletin, vol. 14, no. 1, pp. 23-43, 1984.

[2] S. Asmussen, "Risk theory in a Markovian environment," Scandinavian Actuarial Journal, vol. 1989, no. 2, pp. 69-100, 1989.

[3] A. C. Y. Ng and H. Yang, "On the joint distribution of surplus before and after ruin under a Markovian regime switching model," Stochastic Processes and their Applications, vol. 116, no. 2, pp. 244-266, 2006.

[4] S. M. Li and Y. Lu, "Moments of the dividend payments and related problems in a Markov-modulated risk model," North American Actuarial Journal, vol. 11, no. 2, pp. 65-76, 2007.

[5] Y. Lu and S. M. Li, “The Markovian regime-switching risk model with a threshold dividend strategy," Insurance, vol. 44, no. 2, pp. 296-303, 2009. 
[6] X. Zhang, "On the ruin problem in a Markov-modulated risk model," Methodology and Computing in Applied Probability, vol. 10, no. 2, pp. 225-238, 2008.

[7] J. X. Zhu and H. L. Yang, "Ruin theory for a Markov regimeswitching model under a threshold dividend strategy," Insurance, vol. 42, no. 1, pp. 311-318, 2008.

[8] J. X. Zhu and H. L. Yang, "On differentiability of ruin functions under Markov-modulated models," Stochastic Processes and their Applications, vol. 119, no. 5, pp. 1673-1695, 2009.

[9] W. G. Yu, "A m-type risk model with markov-modulated premium rate," Journal of Applied Mathematics and Informatics, vol. 27, no. 5-6, pp. 1033-1047, 2009.

[10] H.-L. Dong, Z.-T. Hou, and X.-N. Zhang, "The probability of ruin in a kind of Markov-modulated risk model," Chinese Journal of Engineering Mathematics, vol. 26, no. 3, pp. 381-388, 2009.

[11] J. Q. Wei, H. L. Yang, and R. M. Wang, "Optimal threshold dividend strategies under the compound poisson model with regime switching," Stochastic Analysis With Financial Applications, vol. 65, pp. 413-429, 2011.

[12] R. J. Elliott, T. K. Siu, and H. L. Yang, "Ruin theory in a hidden Markov-modulated risk model," Stochastic Models, vol. 27, no. 3, pp. 474-489, 2011.

[13] X.-M. Ma, K. Luo, G.-M. Wang, and Y.-J. Hu, "Constant barrier strategies in a two-state Markov-modulated dual risk model," Acta Mathematicae Applicatae Sinica-English Series, vol. 27, no. 4, pp. 679-690, 2011.

[14] J. G. Dong and G. X. Liu, "Joint distribution of the supremum, infimum and number of zeros in the Markov-modulated risk model," Chinese Journal of Applied Probability and Statistics, vol. 27, no. 5, pp. 473-480, 2011.

[15] X. Y. Mo and X. Q. Yang, "Path-depict and probabilistic construction of the markov-modulated risk model," Acta Mathematicae Applicatae Sinica-Chinese Series, vol. 35, no. 3, pp. 385-395, 2012.

[16] X. Zhang and T. K. Siu, "On optimal proportional reinsurance and investment in a Markovian regime-switching economy," Acta Mathematica Sinica-English Series, vol. 28, no. 1, pp. 6782, 2012.

[17] S. M. Li and J. D. Ren, "The maximum severity of ruin in a perturbed risk process with Markovian arrivals," Statistics and Probability Letters, 2013.

[18] Z. S. Ouyang and Y. Yan, "Some moment's results of present value function of increasing life insurance under random interest rate," Mathematics in Economics, vol. 20, no. 1, pp. 41-47, 2003.

[19] J. Cai, "Ruin probabilities and penalty functions with stochastic rates of interest," Stochastic Processes and their Applications, vol. 112, no. 1, pp. 53-78, 2004.

[20] X. Zhao and J. E. Liu, "A ruin problem for classical risk processes under random interest force," Applied Mathematics, vol. 20, no. 3, pp. 313-319, 2005.

[21] X. Zhao, B. Zhang, and Z. Mao, "Optimal dividend payment strategy under Stochastic Interest Force," Quality and Quantity, vol. 41, no. 6, pp. 927-936, 2007.

[22] J. Z. Li and R. Wu, "Upper bounds for ruin probabilities under stochastic interest rate and optimal investment strategies," Acta Mathematica Sinica-English Series, vol. 28, no. 7, pp. 1421-1430, 2012.
[23] B. Zhang and X. Zhao, "The expected discount penalty function under stochastic interest governed by Markov switching process," Dynamics of Continuous, Discrete \& Impulsive Systems A, vol. 14, pp. 343-348, 2007.

[24] J.-H. Xie and W. Zou, "Expected present value of total dividends in a delayed claims risk model under stochastic interest rates," Insurance, vol. 46, no. 2, pp. 415-422, 2010.

[25] D. J. Yao, R. M. Wang, and L. Xu, "On the expected discounted penalty function associated with the time of ruin for a risk model with random income," Chinese Journal of Applied Probability and Statistics, vol. 24, no. 3, pp. 319-326, 2008. 


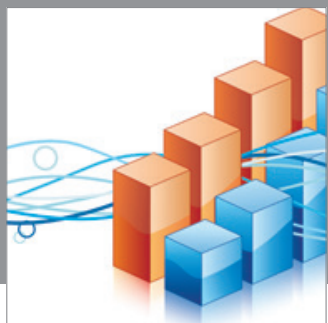

Advances in

Operations Research

mansans

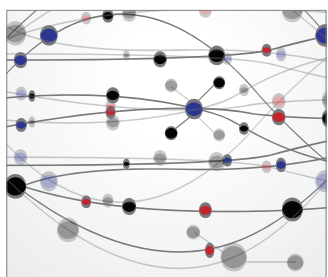

The Scientific World Journal
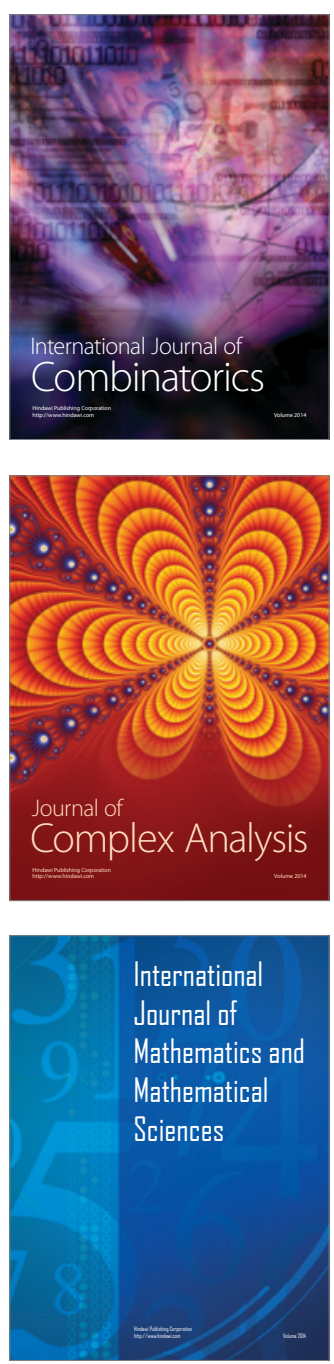
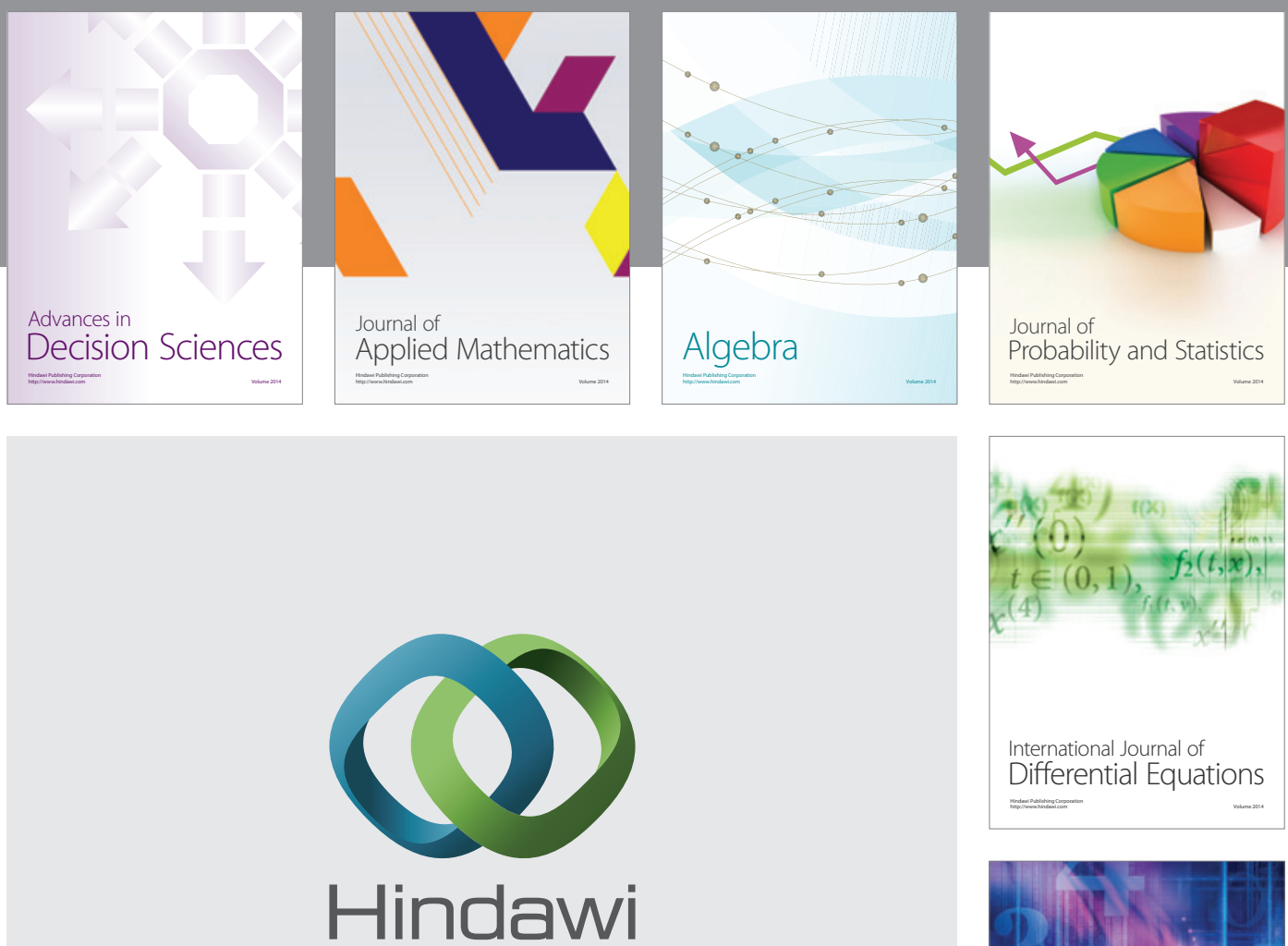

Submit your manuscripts at http://www.hindawi.com
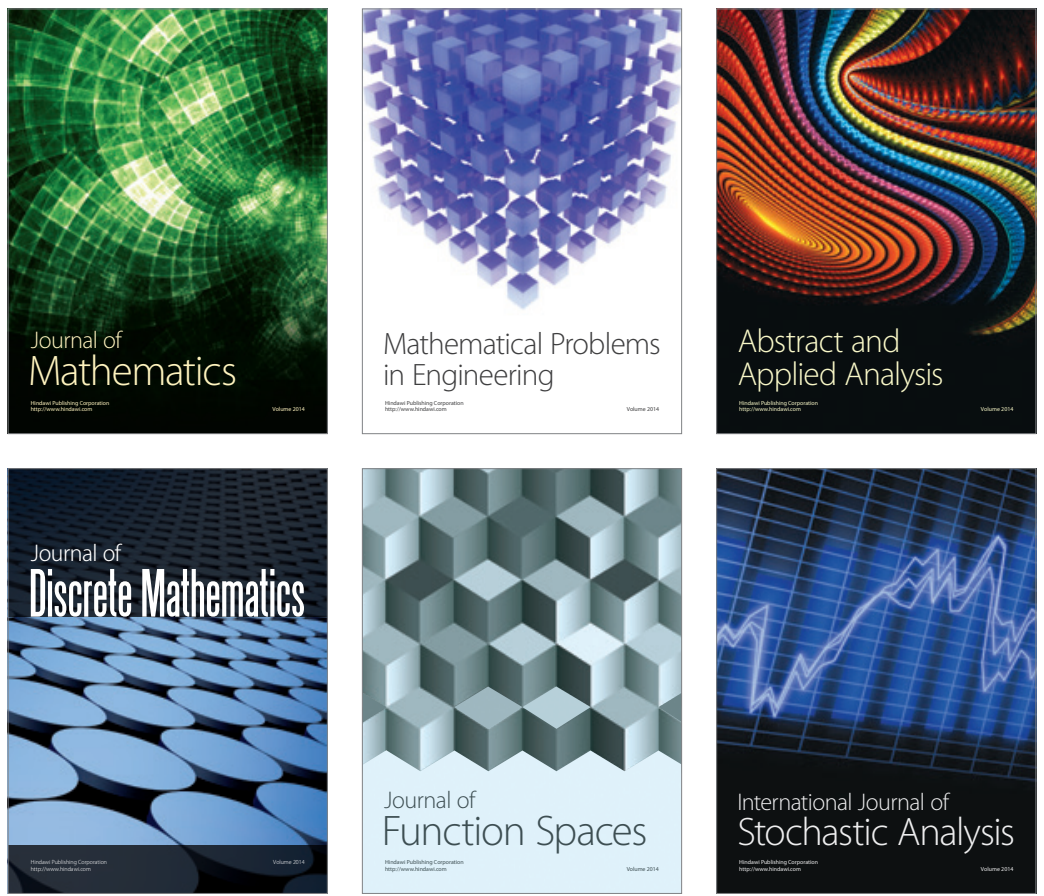

Journal of

Function Spaces

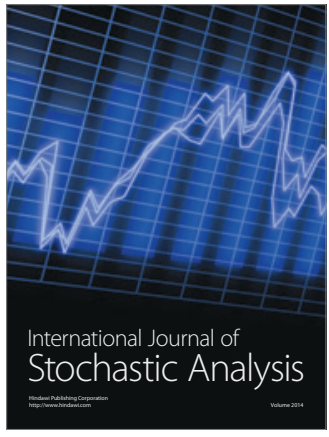

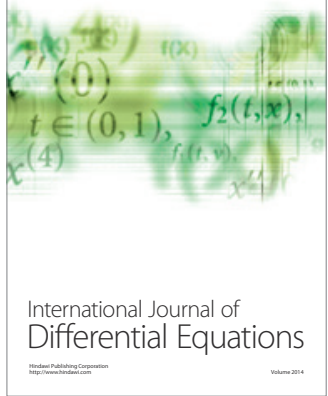
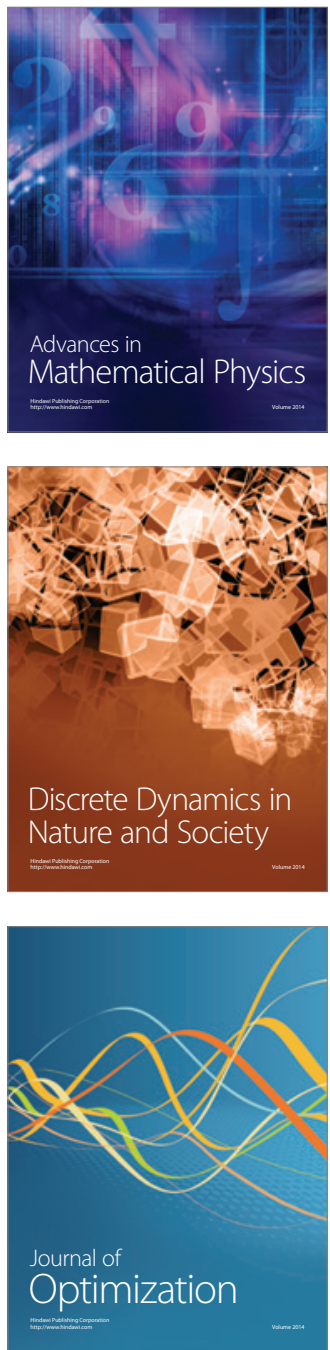